\title{
Advances in the diagnosis of pulmonary tuberculosis in children
}

\author{
Mark P. Nicol, PhD ${ }^{1}$, Heather J Zar, PhD $^{2}$ \\ 1.Division of Infection and Immunity, School of Biomedical Sciences, University of Western \\ Australia, Perth, Australia \\ 2.Department of Paediatrics and Child Health, and SA-MRC Unit on Child \& Adolescent Health, \\ University of Cape Town and Red Cross War Memorial Children's Hospital, Cape Town, South \\ Africa
}

\begin{abstract}
Major challenges still exist in the accurate diagnosis of tuberculosis in children. Algorithms based on clinical and radiological features remain in widespread use despite poor performance. Newer molecular diagnostics allow for rapid identification of TB and detection of drug-resistance in a subset of children, but lack sensitivity. Molecular testing of multiple specimens, including nontraditional specimen types, such as nasopharyngeal aspirates and stool and urine, may improve sensitivity, but the optimal combination of specimens requires further research. Novel tests under development or evaluation include a urine lipoarabinomannan test with improved sensitivity and a range of biomarkers measured from stimulated or unstimulated peripheral blood.
\end{abstract}

\section{Keywords}

tuberculosis; diagnosis; children; Xpert; LAM; biomarker

\section{Limitations of current diagnostic approaches}

The substantial gap between tuberculosis (TB) notifications in children and the estimated true burden of illness(1) may, in part, be due to lack of awareness and screening, but likely also reflects the difficulty in establishing a confirmed diagnosis of pulmonary (PTB) in children. Most PTB diagnoses in children globally are made on clinical and radiological evidence, and microbiological confirmation remains unusual(1).

The reasons for the difficulty in confirming a diagnosis of PTB in children are welldescribed $(2,3)$. Both clinical features and radiological findings are frequently non-specific or subtle, particularly in children with less severe illness $(4,5)$. Microbiological confirmation is hampered by difficulty obtaining samples from the site of disease: young children with

Corresponding address: Mark Nicol: M504, Faculty of Health Sciences, University of Western Australia, Perth, Australia. Telephone: +61 85457 4872. Mark.Nicol@uwa.edu.au.

Publisher's Disclaimer: This is a PDF file of an unedited manuscript that has been accepted for publication. As a service to our customers we are providing this early version of the manuscript. The manuscript will undergo copyediting, typesetting, and review of the resulting proof before it is published in its final form. Please note that during the production process errors may be discovered which could affect the content, and all legal disclaimers that apply to the journal pertain. 
PTB are seldom able to provide an expectorated sputum sample and sputum induction or other sampling is needed which may not be available in high burden settings. Once a sample has been obtained, microbiological testing, whether by culture or nucleic acid detection, is relatively insensitive, compared with adult TB, since TB in children is frequently paucibacillary(6).

The difficulties in confirming a diagnosis of PTB also impact on our ability to assess the accuracy of novel diagnostics. Since microbiological confirmation is only achieved in a proportion of children who are clinically diagnosed with TB, a new diagnostic with better sensitivity than current reference standard microbiological testing, would appear to be poorly specific. As an alternative, a composite reference standard incorporating both microbiologically-confirmed and clinically-diagnosed TB may be used to assess the accuracy of a new test(7). However, the need for new diagnostics is predicated on the assumption that clinical diagnosis lacks accuracy, and so the use of a composite reference standard is an unsatisfactory alternative. New approaches are needed to assess the accuracy of novel diagnostics which perform better than current reference standard methods.

However, there have been several important advances in the past decade. Advances in imaging include the use of chest and mediastinal ultrasound, as well as abdominal ultrasound to detect disseminated disease (Ref. Article on Imaging by T. Pillay in this series). Chest MRI, which shows promise for detecting TB-specific lymphadenopathy may be helpful to develop an improved reference standard, but is not suitable for routine use in diagnosis, due to equipment and expertise required. Testing of alternative, non-invasive specimens, including induced sputum(8), nasopharyngeal aspirates(9), oral swabs(10), stool(11) and urine(12), has demonstrated utility or holds promise. Automated nucleic acid amplification tests provide rapid diagnosis in settings where mycobacterial culture is not feasible(13). Research on biomarkers for rapid identification of children at highest risk is advancing steadily. Several large consortia are now evaluating novel diagnostic approaches in multicentre studies, such as TB-SPEED (https://www.tb-speed.com) and RaPAED(14). Here, we review these advances, consider their role in diagnostics algorithms and highlight future research needed to advance this field.

\section{Diagnostic approaches differ by setting}

Diagnostic algorithms and approaches vary substantially depending on the level of care, resource availability and prevalence of TB. For example, in a well-resourced, low-prevalence setting, children presenting with respiratory symptoms compatible with TB may be given an initial course of antibiotics; lack of response might indicate chest radiography and blood testing for inflammatory markers such as C-reactive protein or complete blood count. A history of recent travel to an endemic area or close contact with a patient with TB would trigger further investigation, including tests of TB-sensitization (tuberculin skin test [TST] or interferon-gamma release assay), additional imaging (such as computed tomography, if chest radiography identifies abnormalities), and collection of respiratory specimens (induced sputum, bronchoalveolar lavage) for mycobacterial culture and automated nucleic acid amplification testing. 
In contrast, in a high burden setting, where the index of suspicion and risk of progression of disease is higher, initial evaluation of the child might include TST and chest radiography. If capacity exists, specimen collection might include induced sputum sampling for automated nucleic acid amplification testing, and liquid mycobacterial culture, if available. Empiric treatment of a child suspected to have TB, in the absence of microbiological confirmation, is more likely in this setting, than in a well-resourced setting, where a clinician may choose to monitor a less-ill child while waiting for microbiological test results.

\section{Advances in the clinical diagnosis of TB in children}

Clinical symptoms in children are non-specific, and clinical scoring systems perform with wide variability.(15) Symptom scores encompass clinical features, chest radiology and tuberculin skin testing. Clinical diagnosis may be especially challenging in HIV-infected, malnourished or young children. While TB was previously regarded as a chronic disease in children with the key clinical features being chronic cough or loss of weight in the preceding 2 to 3 months, it is increasingly recognised that TB occurs in the context of acute pneumonia in children living in high TB prevalence areas. Several African studies have now identified M. tuberculosis from respiratory samples in children with acute pneumonia, with a metaanalysis reporting culture confirmed disease in $8 \%$ of children with pneumonia.(16) Further, in a South African birth cohort study that prospectively investigated children for pneumonia and for TB, TB was strongly associated with recurrent pneumonia in the first 5 years of life. (17) Importantly in this cohort almost all children were HIV negative, although a quarter of mothers were HIV-infected. The strong association of TB with childhood pneumonia, highlights the importance of accurate diagnosis to ensure appropriate therapy, underscoring the need to strengthen diagnosis and integrate childhood TB and pneumonia programs. Advances in radiological diagnosis are addressed in a separate paper in this series, but chest radiography remains the most widely used imaging tool, although specificity and interreader agreement are poor (Ref. Article on Imaging by T. Pillay in this series).

\section{Microscopy and culture for the diagnosis of TB in children}

Smear microscopy is an insensitive test for TB in children. A recent meta-analysis of 15 paediatric studies which compared standard microbiological testing to nucleic acid amplification testing with Xpert MTB/RIF, found the pooled sensitivity of smear microscopy to be $22 \%$ (gastric aspirate) or $29 \%$ (induced/expectorated sputum) compared with mycobacterial culture(6). Mycobacterial culture remains the most sensitive method for microbiological confirmation of TB in children, but since many children are diagnosed clinically with PTB, despite negative microbiological testing, culture is considered to be an imperfect reference standard. For example, in the same meta-analysis, the yield of mycobacterial culture amongst 939 children with a diagnosis of TB was only $24.4 \%(6)$. The apparent low yield of culture may reflect the insensitivity of culture, the poor specificity of clinical diagnosis, or - most likely - the combination of both.

It is perhaps insufficiently recognized that mycobacterial culture is not uniformly wellimplemented. Although automated liquid culture systems are now widely used, and significantly more sensitive than culture on solid media, the process of preparing a sample 
for culture is technically demanding. Potentially contaminated samples, such as sputum, undergo decontamination to remove rapidly growing bacteria, typically with sodium hydroxide, before culture. The concentration of sodium hydroxide used varies by laboratory. Whilst this has not been well-studied, it is possible that less heavily contaminated samples from children may require less harsh decontamination protocols than those from adults, and that this may affect mycobacterial yield. In one before-and-after study, the change from $1.5 \%$ to $1 \%$ sodium hydroxide concentration was associated with an increase in $M$. tuberculosis yield from 6.0 to $9.7 \%$ with no corresponding increase in contamination rate(18). The optimization of mycobacterial culture methods for paediatric samples, and for different respiratory sample types, requires further study.

\section{Nucleic acid amplification tests for the diagnosis of TB in children}

The development and implementation of automated nucleic acid amplification tests (NAATs) for TB has been a significant advance(13). Xpert MTB/RIF has led the field(19), however the BD MAX MDR-TB assay(20) from Becton Dickinson, has recently demonstrated similar accuracy to Xpert MTB/RIF in clinical testing. There are several important advantages to these tests: both are simultaneously able to detect the presence of M. tuberculosis DNA and identify resistance to rifampicin (the BD MAX assay also identifies most cases with isoniazid resistance), tests are rapid, operator dependence is greatly reduced compared to earlier NAATs, and the Xpert MTB/RIF platform is relatively small, and suitable for testing at decentralized sites, closer to the point-of-care(19). A metaanalysis of the accuracy of a single Xpert MTB/RIF test in children found a pooled sensitivity of $62 \%$ and $66 \%$ for sputum and gastric aspirate/lavage respectively, compared with a microbiological reference standard. Specificity for both sample types was $98 \%$ (21). The test identified the presence of $M$. tuberculosis DNA in only $2 \%$ of clinically diagnosed (but culture-negative) cases. Testing an additional sample with Xpert MTB/RIF increased the diagnostic yield (over a single Xpert MTB/RIF test) by between 8 and 20\%.

A newer version of the Xpert MTB/RIF assay, Xpert MTB/RIF Ultra (Ultra) has improved sensitivity (with slightly reduced specificity) in adults(22). Ultra, done on induced or expectorated sputum or bronchoalveolar lavage fluid, has recently been evaluated in several paediatric cohorts where it showed improvement in sensitivity over Xpert MTB/RIF of between $2 \%$ and $10 \%$, with marginally lower specificity(23-25).

The extent of uptake of NAAT testing for TB in children in high burden settings is not wellstudied, however it is likely that cost and the perceived difficulty in obtaining suitable specimens for testing are significant obstacles to wider implementation. Preferential pricing for the Xpert assays in high burden countries is approximately US $\$ 10$ per test, excluding instrument and overhead costs. However, the number of tests for paediatric TB diagnosis is likely only a very small proportion of those done to diagnose adult disease. This reflects the perceived difficulty in obtaining respiratory samples for testing from children. The utility of more accessible samples for TB testing in children has therefore been a major focus of recent research. 


\section{Alternative specimen types for microbiological testing for TB in children}

Several studies have compared the microbiological yield (culture or Xpert MTB/RIF) from induced sputum samples with that from gastric aspirate or lavage. Results are conflicting, with some studies showing improved yield from induced $\operatorname{sputum}(8,26)$, others from gastric aspirate/lavage $(27,28)$ and others no difference $(29)$. It is possible that this variation may reflect familiarity and expertise with collection of particular specimen types. More recently, nasopharyngeal sampling (aspirate or swab) has been described as a promising alternative to more invasive, or resource-intensive, sampling(9). In most studies, the microbiological yield from nasopharyngeal samples was lower than that from gastric aspirate or induced sputum(9, 30-32). Oral swabs collected from the buccal or tongue mucosa are even simpler to collect. A pilot study, using an in-house NAAT, has demonstrated proof of principle, however sensitivity was low(10). The optimization and accuracy of oral swab testing using commercial NAATs is an area requiring further research.

Testing of combinations of different specimens is likely to optimize microbiological yield but may be less feasible and add significant cost. For example, one study in South Africa showed that the combined yield from Xpert MTB/RIF Ultra testing of one induced sputum specimen and two nasopharyngeal aspirates was $88 \%$ (compared to mycobacterial culture of sputum)(33). An alternative to testing multiple specimens individually is to pool specimens and do a single test on the pooled specimen. However, testing of a pooled gastric aspirate, nasopharyngeal aspirate and induced sputum specimen with either culture or Xpert MTB/RIF did not show significant benefit over testing a gastric aspirate alone(34).

There is increasing interest in the use of stool as a diagnostic specimen(11, 35-37). Since young children swallow their sputum, the presence of $M$. tuberculosis in stool may signify either pulmonary or extrapulmonary (gastrointestinal) TB. A recent meta-analysis of the accuracy of Xpert MTB/RIF testing of stool from children with presumptive TB showed a pooled sensitivity of $67 \%$ and specificity of $99 \%$ compared to a microbiological reference standard(11). Sensitivity was higher (79\%) amongst children with HIV infection compared to HIV-uninfected children (60\%). Protocols for the preparation of stool prior to Xpert testing vary widely, and several multicentre studies are currently comparing the yield and feasibility of different stool processing methods.

In summary, testing of a range of respiratory or non-respiratory samples with Xpert MTB/RIF or Ultra allows rapid identification of children with TB, as well as rifampicin susceptibility. Repeated tests ( 2 specimens per child) in children substantially improve sensitivity. Since test specificity is high, a positive test can be used to rule-in TB in a child, except where the pre-test probability of TB is very low or where a child has been recently treated for TB, as the test may remain positive for months(38). However, since sensitivity, even with multiple specimens, remains lower than that of culture, NAAT testing should not be used to rule out a diagnosis of TB in a child.

Urine can be relatively easily obtained from children and is therefore an attractive specimen for TB testing. Mycobacterial lipoarabinomannan (LAM) can be detected in the urine of adult TB patients with advanced HIV infection using a simple lateral flow assay, suitable for 
use at the point-of-care(39). Randomized clinical trials have shown that implementation of the Alere Determine TB LAM test (AlereLAM, Abbott, Chicago, IL, USA) for rapid diagnosis of HIV-associated TB reduces mortality amongst adult in-patients $(40,41)$. The reported sensitivity of AlereLAM for diagnosis of TB in HIV-infected children has ranged from $43 \%$ to $65 \%$, with a very wide range in specificity (51\% to $94 \%)(42-44)$. The reasons for the wide range in specificity are not clear, but may relate to the patient population, reference standard testing, or, most probably, specimen collection method. Although method of specimen collection is not always reported, in younger children the use of urine bags may be associated with heavy contamination by skin and faecal flora. Contamination with bacteria found in stool may result in false-positive LAM tests(45). More recently, Broger and colleagues have reported on the accuracy of a novel LAM assay, Fujifilm SILVAMP TB LAM (FujiLAM; Fujifilm, Tokyo, Japan)(46), which showed substantially improved sensitivity in HIV-infected adults compared with AlereLAM (70\% vs. $42 \%)$. The results of the first evaluations of FujiLAM in children are expected soon.

\section{Host biomarkers for the diagnosis of TB in children}

Whilst microbiological diagnosis aims to detect the causative pathogen, diagnostic approaches using host biomarkers aim to identify a characteristic immune or inflammatory response to $M$. tuberculosis. Biomarkers may be measured from blood samples tested directly ex vivo, or from samples which are first stimulated with mycobacterial antigens, to elicit M. tuberculosis-specific responses. Commercially-available interferon-gamma release assays are based on the latter principle, and, while specific for the pathogen, are unable to distinguish between active infection, recent prior infection and latent infection(47). Other approaches using stimulated blood include ELISPOT (Enzyme-linked enzyme-Linked Immune Absorbent Spot) assays, targeting T helper 1 cytokines such as interferon-gamma and interleukin-2(48), the detection of antibodies secreted by TB-specific plasma cells(49)and flow cytometry-based tests, such as the T cell activation marker (TAM)-TB assay(50). The TAM-TB assay has shown promising accuracy in a small study (sensitivity $83 \%$, specificity $97 \%$ in 113 children of whom 18 had culture-confirmed TB), but is currently complex and not suitable for implementation outside of research settings.

However, assays based on direct ex vivo testing of blood are most attractive, as they hold the potential for inexpensive testing close to the point of care. These may be based on detection of metabolic markers(51) or, most promisingly, of host transcriptional signatures. A large multicentre African study by Anderson and colleagues(7) used microarrays to identify a 51transcript RNA signature from children in South Africa and Malawi, and then validated the findings in a cohort of Kenyan children. In the validation cohort, sensitivity and specificity were $83 \%$ and $84 \%$ for microbiologically-proven TB, and a disease risk score derived from the transcriptional profile showed a strong relationship with strength of diagnosis amongst children with clinically diagnosed TB. There are several major hurdles which tests of this type will need to overcome before translation into clinical use, including further validation in different populations, reduction of the number of transcripts required to identify TB, transfer to a testing platform more suitable for testing in hospital laboratories and field evaluation in the setting of intended use. However, technologies are rapidly evolving, and these obstacles are not insurmountable. 
Despite these important advances, a major obstacle remains to the evaluation and implementation of diagnostics for TB in children. This is well-illustrated by the Anderson biomarker study. In this study, the transcript-based disease risk score was positive in $63 \%$ of those in whom tuberculosis was 'highly probable', $42 \%$ of those in whom it was "probable, and $35 \%$ of those in whom it was "possible". Since these positive scores were found in children with negative microbiological tests for $\mathrm{TB}$, it is impossible to obtain a true estimate of the accuracy of the transcript-based score. Statistical approaches, such as latent class analysis(52), may assist in addressing this difficulty, however the only way to convincingly demonstrate the accuracy of a novel diagnostic with improved sensitivity over the current reference standard of mycobacterial culture, is through interventional studies. The clinical evaluation of a novel, highly-sensitive diagnostic, for example, a biomarker with very high sensitivity which could be used as a rule-out test for TB, will inevitably require implementation in a diagnostic algorithm in which treatment decisions are made, at least in part, on the index test being evaluated. These studies will need to be done in highly controlled clinical settings with close follow up of children, but if we are to make real advances in the development of improved diagnostics for TB in children, such studies are essential.

\section{Acknowledgments}

Declarations of Interest: The authors have received grant funding for paediatric TB diagnostic evaluation (Xpert MTB/RIF, Xpert MTB/RIF Ultra, AlereLAM, FujiLAM) from Regional Prospective Observational Research in Tuberculosis (RePORT TB) Consortium which is co-funded by the Medical Research Council of South Africa and the US Office of AIDS Research of the National Institutes of Health of the USA (DAA2-16-62066-1), the Medical Research Council of South Africa for the Tuberculosis Collaborating Centre for Child Health and for the MRC Unit on Child and Adolescent Health and from the NIH (RO1HD058971).

\section{References}

1. Dodd PJ, Gardiner E, Coghlan R, Seddon JA. Burden of childhood tuberculosis in 22 high-burden countries: a mathematical modelling study. The Lancet Global health. 2014;2(8):e453-9. [PubMed: 25103518]

2. Nicol MP, Zar HJ. New specimens and laboratory diagnostics for childhood pulmonary TB: progress and prospects. Paediatr Respir Rev. 2011;12(1):16-21. [PubMed: 21172670]

3. Reuter A, Hughes J, Furin J. Challenges and controversies in childhood tuberculosis. Lancet. 2019;394(10202):967-78. [PubMed: 31526740]

4. Hatherill M, Hanslo M, Hawkridge T, Little F, Workman L, Mahomed H, et al. Structured approaches for the screening and diagnosis of childhood tuberculosis in a high prevalence region of South Africa. Bull World Health Organ. 2010;88(4):312-20. [PubMed: 20431796]

5. Graham SM, Cuevas LE, Jean-Philippe P, Browning R, Casenghi M, Detjen AK, et al. Clinical Case Definitions for Classification of Intrathoracic Tuberculosis in Children: An Update. Clinical infectious diseases : an official publication of the Infectious Diseases Society of America. 2015;61Suppl 3:S179-87.

6. DiNardo AR, Detjen A, Ustero P, Ngo K, Bacha J, Mandalakas AM. Culture is an imperfect and heterogeneous reference standard in pediatric tuberculosis. Tuberculosis (Edinb). 2016;101S:S105S8. [PubMed: 27727131]

7. Anderson ST, Kaforou M, Brent AJ, Wright VJ, Banwell CM, Chagaluka G, et al. Diagnosis of childhood TB in High TB/HIV burden African countries by host RNA expression. New England Journal of Medicine. 2014; In press. 
8. Zar HJ, Hanslo D, Apolles P, Swingler G, Hussey G. Induced sputum versus gastric lavage for microbiological confirmation of pulmonary tuberculosis in infants and young children: a prospective study. Lancet. 2005;365(9454):130-4. [PubMed: 15639294]

9. Zar HJ, Workman L, Isaacs W, Munro J, Black F, Eley B, et al. Rapid molecular diagnosis of pulmonary tuberculosis in children using nasopharyngeal specimens. Clinical infectious diseases : an official publication of the Infectious Diseases Society of America. 2012;55(8):1088-95. [PubMed: 22752518]

10. Nicol MP, Wood RC, Workman L, Prins M, Whitman C, Ghebrekristos Y, et al. Microbiological diagnosis of pulmonary tuberculosis in children by oral swab polymerase chain reaction. Sci Rep. 2019;9(1):10789. [PubMed: 31346252]

11. MacLean E, Sulis G, Denkinger CM, Johnston JC, Pai M, Ahmad Khan F. Diagnostic Accuracy of Stool Xpert MTB/RIF for Detection of Pulmonary Tuberculosis in Children: a Systematic Review and Meta-analysis. J Clin Microbiol. 2019;57(6).

12. Nicol MP, Allen V, Workman L, Isaacs W, Munro J, Pienaar S, et al. Urine lipoarabinomannan testing for diagnosis of pulmonary tuberculosis in children: a prospective study. Lancet Glob Health. 2014;2(5):e278-84. [PubMed: 24818083]

13. Nicol MP, Workman L, Isaacs W, Munro J, Black F, Eley B, et al. Accuracy of the Xpert MTB/RIF test for the diagnosis of pulmonary tuberculosis in children admitted to hospital in Cape Town, South Africa: a descriptive study. The Lancet Infectious diseases. 2011;11(11):819-24. [PubMed: 21764384]

14. Olbrich L, Zar H, Graham S, Sabi I, Ntinginya NE, Bhatt N, et al. OC 8561 RAPAED TB: AN INNOVATIVE CHILD TB DIAGNOSTIC VALIDATION STUDY. BMJ Global Health. 2019;4:A13-A.

15. Pearce EC, Woodward JF, Nyandiko WM, Vreeman RC, Ayaya SO. A systematic review of clinical diagnostic systems used in the diagnosis of tuberculosis in children. AIDS Res Treat. 2012;2012:401896. [PubMed: 22848799]

16. Oliwa JN, Karumbi JM, Marais BJ, Madhi SA, Graham SM. Tuberculosis as a cause or comorbidity of childhood pneumonia in tuberculosis-endemic areas: a systematic review. The Lancet Respiratory medicine. 2015;3(3):235-43. [PubMed: 25648115]

17. Martinez L, le Roux DM, Barnett W, Stadler A, Nicol MP, Zar HJ. Tuberculin skin test conversion and primary progressive tuberculosis disease in the first 5 years of life: a birth cohort study from Cape Town, South Africa. Lancet Child Adolesc Health. 2018;2(1):46-55. [PubMed: 29457055]

18. Whitelaw AC, Mentoor K, Zar HJ, Nicol MP, editor Standardmycobacterial decontamination protocols may be inappropriate for samples from paediatric patients. 41st World Conference on Lung Health of the International Union Against Tuberculosis and Lung Disease (The Union); 2010; Berlin. Paris2010.

19. Lawn SD, Nicol MP. Xpert(R) MTB/RIF assay: development, evaluation and implementation of a new rapid molecular diagnostic for tuberculosis and rifampicin resistance. Future microbiology. 2011;6(9):1067-82. [PubMed: 21958145]

20. Shah M, Paradis S, Betz J, Beylis N, Bharadwaj R, Caceres T, et al. Multicenter Study of the Accuracy of the BD MAX MDR-TB Assay for Detection of Mycobacterium tuberculosis Complex and Mutations Associated with Resistance to Rifampin and Isoniazid. Clin Infect Dis. 2019.

21. Detjen AK, DiNardo AR, Leyden J, Steingart KR, Menzies D, Schiller I, et al. Xpert MTB/RIF assay for the diagnosis of pulmonary tuberculosis in children: a systematic review and metaanalysis. The Lancet Respiratory medicine. 2015;3(6):451-61. [PubMed: 25812968]

22. Dorman SE, Schumacher SG, Alland D, Nabeta P, Armstrong DT, King B, et al. Xpert MTB/RIF Ultra for detection of Mycobacterium tuberculosis and rifampicin resistance: a prospective multicentre diagnostic accuracy study. The Lancet Infectious diseases. 2018;18(1):76-84. [PubMed: 29198911]

23. Nicol MP, Workman L, Prins M, Bateman L, Ghebrekristos Y, Mbhele S, et al. Accuracy of Xpert $\mathrm{Mtb} /$ Rif Ultra for the Diagnosis of Pulmonary Tuberculosis in Children. The Pediatric infectious disease journal. 2018;37(10):e261-e3. [PubMed: 29474257]

24. Sun L, Qi X, Liu F, Wu X, Yin Q, Guo Y, et al. A Test for More Accurate Diagnosis of Pulmonary Tuberculosis. Pediatrics. 2019;144(5). 
25. Sabi I, Rachow A, Mapamba D, Clowes P, Ntinginya NE, Sasamalo M, et al. Xpert MTB/RIF Ultra assay for the diagnosis of pulmonary tuberculosis in children: a multicentre comparative accuracy study. J Infect. 2018;77(4):321-7. [PubMed: 30036606]

26. Qureshi UA, Gupta AK, Mahajan B, Qurieshi MA, Altaf U, Parihar R, et al. Microbiological diagnosis of pulmonary tuberculosis in children: comparative study of induced sputum and gastric lavage. Indian J Pediatr. 2011;78(11):1429-30. [PubMed: 21614605]

27. Mukherjee A, Singh S, Lodha R, Singh V, Hesseling AC, Grewal HM, et al. Ambulatory gastric lavages provide better yields of Mycobacterium tuberculosis than induced sputum in children with intrathoracic tuberculosis. Pediatr Infect Dis J. 2013;32(12):1313-7. [PubMed: 23958816]

28. Singh S, Singh A, Prajapati S, Kabra SK, Lodha R, Mukherjee A, et al. Xpert MTB/RIF assay can be used on archived gastric aspirate and induced sputum samples for sensitive diagnosis of paediatric tuberculosis. BMC Microbiol. 2015;15:191. [PubMed: 26420261]

29. Hatherill M, Hawkridge T, Zar HJ, Whitelaw A, Tameris M, Workman L, et al. Induced sputum or gastric lavage for community-based diagnosis of childhood pulmonary tuberculosis? Arch Dis Child. 2009;94(3):195-201. [PubMed: 18829621]

30. Owens S, Abdel-Rahman IE, Balyejusa S, Musoke P, Cooke RP, Parry CM, et al. Nasopharyngeal aspiration for diagnosis of pulmonary tuberculosis. Arch Dis Child. 2007;92(8):693-6. [PubMed: 17185437]

31. Oberhelman RA, Soto-Castellares G, Gilman RH, Caviedes L, Castillo ME, Kolevic L, et al. Diagnostic approaches for paediatric tuberculosis by use of different specimen types, culture methods, and PCR: a prospective case-control study. The Lancet Infectious diseases. 2010;10(9):612-20. [PubMed: 20656559]

32. Al-Aghbari N, Al-Sonboli N, Yassin MA, Coulter JB, Atef Z, Al-Eryani A, et al. Multiple sampling in one day to optimize smear microscopy in children with tuberculosis in Yemen. PLoS One. 2009;4(4):e5140. [PubMed: 19357770]

33. Zar HJ, Workman LJ, Prins M, Bateman LJ, Mbhele SP, Whitman CB, et al. Tuberculosis Diagnosis in Children Using Xpert Ultra on Different Respiratory Specimens. Am J Respir Crit Care Med. 2019;200(12):1531-8. [PubMed: 31381861]

34. Walters E, van der Zalm MM, Demers AM, Whitelaw A, Palmer M, Bosch C, et al. Specimen Pooling as a Diagnostic Strategy for Microbiologic Confirmation in Children with Intrathoracic Tuberculosis. Pediatr Infect Dis J. 2019;38(6):e128-e31. [PubMed: 30418355]

35. Nicol MP, Spiers K, Workman L, Isaacs W, Munro J, Black F, et al. Xpert MTB/RIF testing of stool samples for the diagnosis of pulmonary tuberculosis in children. Clinical infectious diseases : an official publication of the Infectious Diseases Society of America. 2013;57(3):e18-21. [PubMed: 23580738]

36. Walters E, Gie RP, Hesseling AC, Friedrich SO, Diacon AH, Gie RP. Rapid Diagnosis of Pediatric Intrathoracic Tuberculosis From Stool Samples Using the Xpert MTB/RIF Assay: A Pilot Study. Pediatr Infect Dis J. 2012;31(12):1316.

37. Wolf H, Mendez M, Gilman RH, Sheen P, Soto G, Velarde AK, et al. Diagnosis of pediatric pulmonary tuberculosis by stool PCR. Am J Trop Med Hyg. 2008;79(6):893-8. [PubMed: 19052299]

38. Theron G, Venter R, Smith L, Esmail A, Randall P, Sood V, et al. False-Positive Xpert MTB/RIF Results in Retested Patients with Previous Tuberculosis: Frequency, Profile, and Prospective Clinical Outcomes. J Clin Microbiol. 2018;56(3).

39. Lawn SD, Dheda K, Kerkhoff AD, Peter JG, Dorman S, Boehme CC, et al. Determine TB-LAM lateral flow urine antigen assay for HIV-associated tuberculosis: recommendations on the design and reporting of clinical studies. BMC infectious diseases. 2013;13:407. [PubMed: 24004840]

40. Gupta-Wright A, Corbett EL, van Oosterhout JJ, Wilson D, Grint D, Alufandika-Moyo M, et al. Rapid urine-based screening for tuberculosis in HIV-positive patients admitted to hospital in Africa (STAMP): a pragmatic, multicentre, parallel-group, double-blind, randomised controlled trial. Lancet. 2018;392(10144):292-301. [PubMed: 30032978]

41. Peter JG, Zijenah LS, Chanda D, Clowes P, Lesosky M, Gina P, et al. Effect on mortality of pointof-care, urine-based lipoarabinomannan testing to guide tuberculosis treatment initiation in HIV- 
positive hospital inpatients: a pragmatic, parallel-group, multicountry, open-label, randomised controlled trial. Lancet. 2016;387(10024):1187-97. [PubMed: 26970721]

42. Nicol M, Allen V, Workman L, Isaacs W, Munro J, Black F, et al. Urine lipoarabinomannan testing for the diagnosis of pulmonary tuberculosis in young children - a prospective study. Lancet Global Health. 2014; In press.

43. Kroidl I, Clowes P, Reither K, Mtafya B, Rojas-Ponce G, Ntinginya EN, et al. Performance of urine lipoarabinomannan assays for paediatric tuberculosis in Tanzania. Eur Respir J. 2015;46(3):761-70. [PubMed: 26113682]

44. LaCourse SM, Pavlinac PB, Cranmer LM, Njuguna IN, Mugo C, Gatimu J, et al. Stool Xpert MTB/RIF and urine lipoarabinomannan for the diagnosis of tuberculosis in hospitalized HIVinfected children. Aids. 2018;32(1):69-78. [PubMed: 29028662]

45. Kroidl I, Clowes P, Mwakyelu J, Maboko L, Kiangi A, Rachow A, et al. Reasons for false-positive lipoarabinomannan ELISA results in a Tanzanian population. Scand J Infect Dis. 2014;46(2):1448. [PubMed: 24274710]

46. Broger T, Sossen B, du Toit E, Kerkhoff AD, Schutz C, Ivanova Reipold E, et al. Novel lipoarabinomannan point-of-care tuberculosis test for people with HIV: a diagnostic accuracy study. Lancet Infect Dis. 2019;19(8):852-61. [PubMed: 31155318]

47. Starke JR, Committee On Infectious D. Interferon-gamma release assays for diagnosis of tuberculosis infection and disease in children. Pediatrics. 2014;134(6):el763-73.

48. Chiappini E, Della Bella C, Bonsignori F, Sollai S, Amedei A, Galli L, et al. Potential role of M. tuberculosis specific IFN-gamma and IL-2 ELISPOT assays in discriminating children with active or latent tuberculosis. PLoS One. 2012;7(9):e46041. [PubMed: 23029377]

49. Raqib R, Mondal D, Karim MA, Chowdhury F, Ahmed S, Luby S, et al. Detection of antibodies secreted from circulating Mycobacterium tuberculosis-specific plasma cells in the diagnosis of pediatric tuberculosis. Clin Vaccine Immunol. 2009;16(4):521-7. [PubMed: 19193833]

50. Portevin D, Moukambi F, Clowes P, Bauer A, Chachage M, Ntinginya NE, et al. Assessment of the novel T-cell activation marker-tuberculosis assay for diagnosis of active tuberculosis in children: a prospective proof-of-concept study. Lancet Infect Dis. 2014;14(10):931-8. [PubMed: 25185458]

51. Sun L, Li JQ, Ren N, Qi H, Dong F, Xiao J, et al. Utility of Novel Plasma Metabolic Markers in the Diagnosis of Pediatric Tuberculosis: A Classification and Regression Tree Analysis Approach. J Proteome Res. 2016;15(9):3118-25. [PubMed: 27451809]

52. Schumacher SG, van Smeden M, Dendukuri N, Joseph L, Nicol MP, Pai M, et al. Diagnostic Test Accuracy in Childhood Pulmonary Tuberculosis: A Bayesian Latent Class Analysis. American journal of epidemiology. 2016;184(9):690-700. [PubMed: 27737841] 


\section{Educational aims:}

The reader will be able:

- To highlight limitations in current diagnostic approaches for TB in children

- To review the role of nucleic acid amplification assays in the diagnosis of TB in children

- To identify specimen types most suitable for microbiological testing for TB in children

- To identify promising new tests in development or under evaluation 
Future directions for research

- The assessment of diagnostic algorithms utilising clinical indicators and different diagnostic testing procedures.

- Investigation of the best readily gathered samples [Saliva, sputum, blood, stool, urine] matched to available technology at point of care for diagnostic testing.

- An evaluation of accurate, reliable and inexpensive testing kits for preferred diagnostic parameters at point of care sites. 